\title{
A simple method using a single video camera to determine the three-dimensional position of a fish
}

\author{
PEDRO PEREIRA \\ Universidade de Lisboa, Cascais, Portugal \\ and \\ RUI F. OLIVEIRA \\ Instituto Superior de Psicologia Aplicada, Lisbon, Portugal
}

\begin{abstract}
In the present paper, we describe a method for recording the coordinates of a fish in an aquarium in a three-dimensional space, using a single video camera and a mirror. We use photogrammetic equations for this, considering the image obtained in the mirror as a virtual image obtained by a second camera. A transformation of the coordinate system is required to express the obtained coordinates in an $x, y, z$ system defined by the edges of the aquarium. The accuracy of the proposed method was estimated, and errors in extreme conditions were found to be $0.8 \%$ to $1.2 \%$, compared with the dimensions of the aquarium used in the test.
\end{abstract}

Pelagic organisms live in a three-dimensional (3-D) aquatic environment. The study of spatial aspects of behavior (e.g., structure of fish schools, foraging patterns, swimming behavior) in these animals requires the knowledge of their exact position in space. Several methods have been developed to obtain the 3-D coordinates of animals, based on two different techniques: shadows and stereophotography. The shadow methods are based on the projection of the fish shadows on the substratum and require special experimental conditions, such as shallow water and a homogeneous bottom pattern (Cullen, Shaw, \& Baldwin, 1965; Dambach, 1963; Partridge, Pitcher, Cullen, \& Wilson, 1980), which, in the majority of the situations, are incompatible with the experimental procedures. The stereoscopic methods are based on two simultaneous images obtained from different positions. For this purpose, different techniques have been developed, such as the use of a stereo prism attached to a camera (Cullen et al., 1965), different arrangements of mirrors (Pitcher, 1973, 1975; Symons, 1971a, 1971b), or the use of a pair of photo or video cameras (Coughlin, Strickler, \& Sanderson, 1992; Dahmen \& Zeil, 1984; Dill, Dunbrack, \& Major, 1981). The objective of the present paper is to illustrate the use of traditional photogrammetry for determining the 3-D coordinates of an animal in a closed environment - in our

We thank Vitor Almada, Pedro Ré, and Lucas Noldus for commenting on the manuscript, and Hunter Halder, who reviewed the English. We would also like to thank two anonymous referees for their constructive criticism. During the preparation of this paper, the authors were supported by a grant from Junta Nacional de Investigação Científica e Tecnológica (JNICT). Address correspondence to R. Oliveira, Instituto Superior de Psicologia Aplicada, R. Jardim do Tabaco 44, P-1 100 Lisboa, Portugal. case, a fish in an aquarium - by using a mirror and a single video camera.

\section{Material and Methods}

In order to use the common photogrammetry formula (Wolf, 1985) to get the true 3-D coordinates of a fish in an aquarium by using a single video camera, we used a mirror placed at the left side of the aquarium. The optical axis of the video camera was set to meet the lateral plane of the aquarium, and the mirror was placed so that it formed an angle of $45^{\circ}$ with the lateral plane of the aquarium (see Figure 1). With this arrangement, it is possible to have two views of the aquarium - the front view in the right side of the image and the lateral view in the left side. Therefore, this arrangement is equivalent to having two video cameras - one in the actual position used, and the other perpendicular to the first, with its distance from the aquarium equivalent to the first, plus the distance between the frontal and rear plane of the aquarium (Figure 1). Since the focus of the camera is fixed to the anterior plane of the aquarium, the images in the mirror, or of the fish near the posterior plane of the aquarium, were a little bit out of focus. We compensated for this by increasing the depth of field.

The 3-D coordinates can be easily estimated by referring to Figure 2. Knowing the aquarium dimensions, the distance from the camera to the aquarium, $O$, and the focal length, $f$, we can calculate the distance, $B$ (baseline), by using the lens formula:

$$
\frac{1}{f}=\frac{1}{o}+\frac{1}{i} \Leftrightarrow \frac{1}{i}=\frac{1}{f}-\frac{1}{o},
$$

where $i$ is the image distance (distance from the lens center to the plane of focus), and by the Pythagorean theorem: 


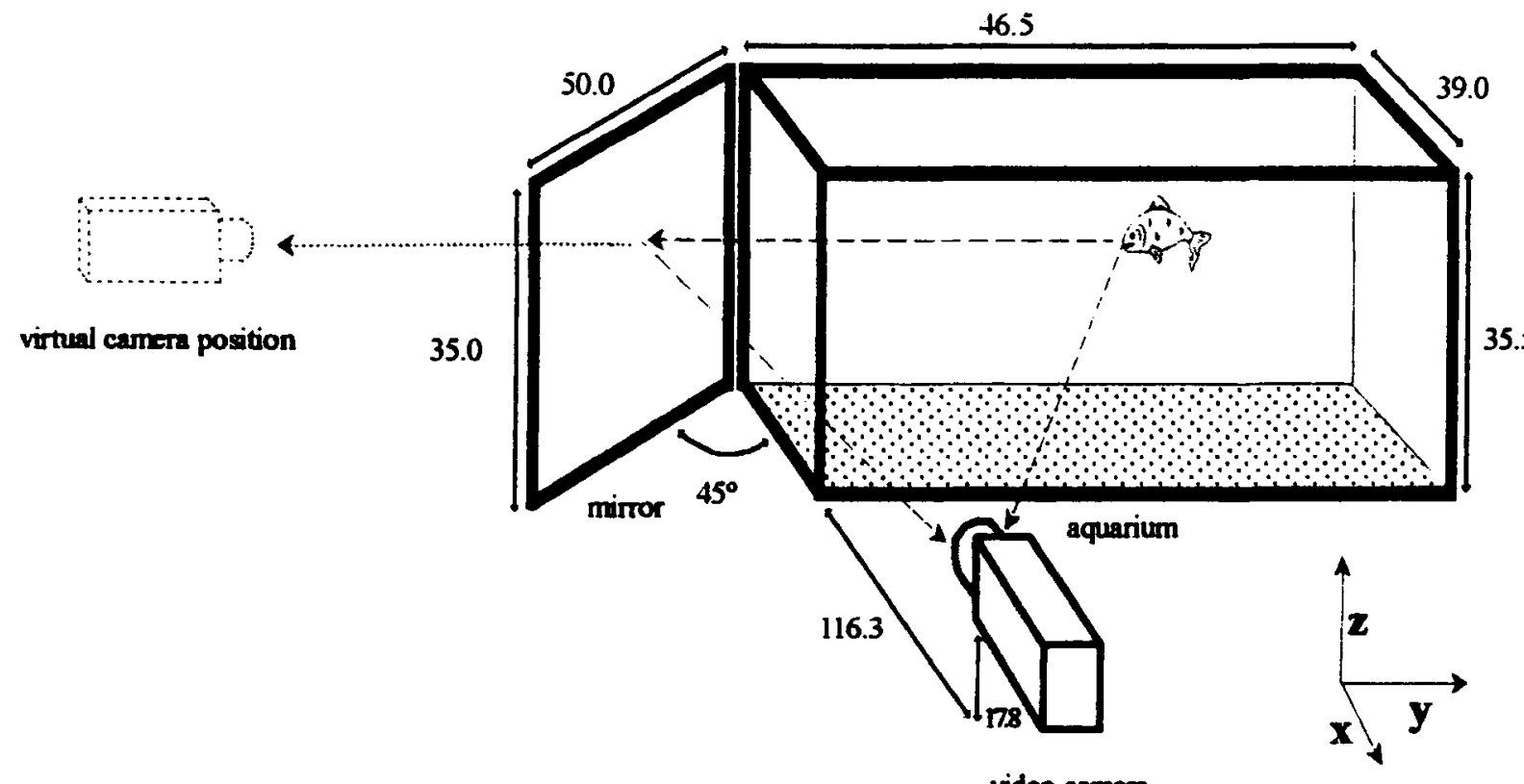

video camera

final coordinate

system

Figure 1. Three-dimensional diagram of the general arrangement of the proposed method (not to scale). All measures are in centimeters. The arrows represent two rays of light, one directed at the camera and one reflected in the mirror.

$$
B=\sqrt{2\left(o+i+\operatorname{depth}_{\mathrm{aq}}\right)^{2}},
$$

where depth $\mathrm{aq}_{\text {is }}$ the depth of the aquarium (distance from the anterior wall to the rear wall of the aquarium). Since the triangle formed by $L, L^{\prime}$, and $O$ is isosceles, the angles $\delta$ and $\delta^{\prime}$ have $45^{\circ}$. The angles $\alpha$ and $\alpha^{\prime}$ may be found by using:

$$
\alpha=\tan ^{-1}\left(x_{\alpha} / f\right) \text { and } \alpha^{\prime}=\tan ^{-1}\left(x_{\alpha^{\prime}} / f\right),
$$

where $x_{a}$ and $x_{\alpha^{\prime}}$ are the positions of the fish in the image (relative to image center), and $f$ is the focal length of the camera lens. Since $x_{a}$ and $x_{a^{\prime}}$ were measured in pixels, $f$ had to be specified in pixels too. Furthermore, because the lens camera was not focused on infinite, the value of $f$ used in the equations was not the nominal focal length of the lens camera. To obtain the value of $f$ in pixels, we used seven points (the visible corners of the aquarium) of known coordinates. We put all the equations below in a spreadsheet, calculated the errors, and then let the Solver substitute the value of $f$, minimizing the errors. We could not calculate the value of $f$ directly from measurements on images, because we did not know the exact dimension of the CCD (charge coupled device) of the camera. Therefore, we did not know how many millimeters on the CCD corresponded to a pixel of the digitized image.

The angles $\phi, \phi^{\prime}$, and $\phi^{\prime \prime}$ were calculated as follows:

$$
\phi=\delta-\alpha
$$

$$
\begin{aligned}
& \phi^{\prime}=\delta^{\prime}+\alpha^{\prime} \\
& \phi^{\prime \prime}=180^{\circ}-\phi-\phi^{\prime}
\end{aligned}
$$

Applying the law of sines, the distances $L A$ and $L^{\prime} A$ may be calculated as:

$$
\begin{aligned}
L A & =B \sin \phi^{\prime} / \sin \phi^{\prime \prime} \\
L^{\prime} A & =B \sin \phi / \sin \phi^{\prime \prime}
\end{aligned}
$$

and the coordinates of the fish will be:

$$
\begin{aligned}
& x_{A}=(L A) \cos \phi \\
& y_{A}=(L A) \sin \phi
\end{aligned}
$$

These coordinates refer to the object-space coordinate system, with origin in $L$ and with the $x$-axis in the plane of the baseline (Figure 2). All of the formulas used so far can be found in Wolf (1985) or in any other book on close-range photogrammetry.

For graphical interpretation of the paths of the fish, we reallocated this coordinate system, placing the origin in the left, posterior corner of the aquarium:

$$
\begin{gathered}
X_{A \mathrm{aq}}=\left(X_{A}-X_{O}\right) \cos \theta-\left(Y_{A}-Y_{O}\right) \sin \theta \\
Y_{A \mathrm{aq}}=\left(X_{A}-X_{O}\right) \sin \theta+\left(Y_{A}-Y_{O}\right) \cos \theta,
\end{gathered}
$$

where $X_{O}$ and $Y_{O}$ are the coordinates of Point $O$ (Figure 2 ) in the initial reference system, and $\theta$ is the angle 


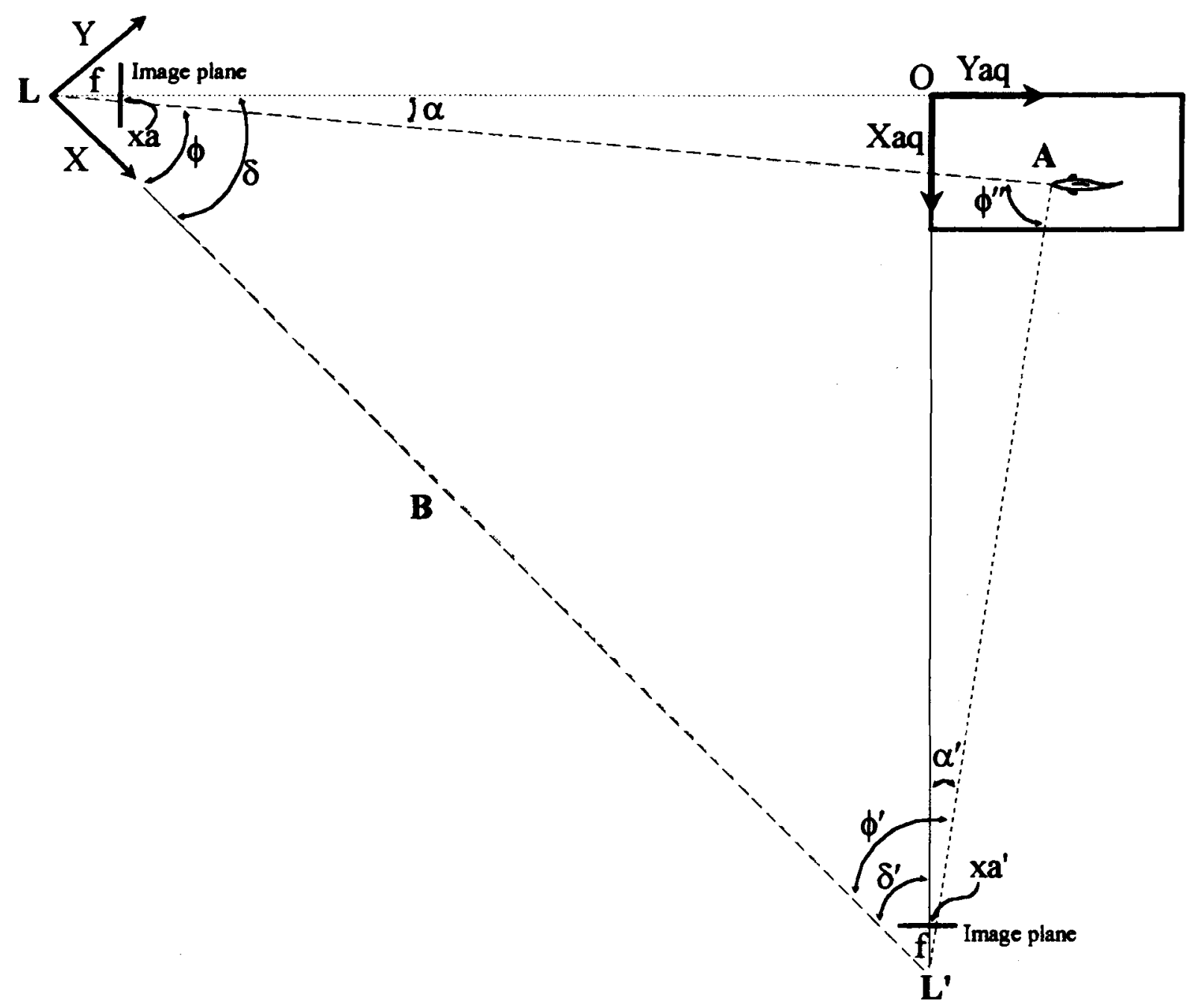

Figure 2. Main distances and angles involved in the calculation of the coordinates of Point $A$, viewed from above. $X_{a}$ and $X_{a^{\prime}}$ are the image coordinates of Point $A$ (see text for details).

of rotation (Harrington, 1987), which was $45^{\circ}$ in this case.

The $z$ coordinate can be determined as:

$$
\begin{aligned}
& V_{A}^{\prime}=\left(L^{\prime} A\right) \tan \beta^{\prime} \\
& Z_{A}=\text { elevation of } L^{\prime}+V_{A}^{\prime},
\end{aligned}
$$

where $L^{\prime} A$ is the distance already found with Equation 1, the elevation of $L^{\prime}$ is the elevation of the camera relative to the base of the aquarium (elevation 0 ), and $\beta$ is calculated by using:

$$
\beta^{\prime}=\tan ^{-1}\left(y_{a^{\prime}} / x_{a^{\prime}}^{2}+f^{2}\right)^{1 / 2},
$$

where $y_{a^{\prime}}$ and $x_{a^{\prime}}$ are the coordinates of the fish in the image, and $f$ is the focal length in pixels, as calculated above (Wolf, 1985).

\section{Results}

The accuracy of the proposed method was estimated by standard deviation and the average of the errors between (1) the calculated and the true positions of 77 benchmarks placed all over the aquarium, and (2) the distances between 65 pairs of these points chosen at random.

A Sony Model V600E color video camera was used. All measurements were taken from digitized images with a Metrabyte MV1A frame grabber with a $512 \times 512$ pixel resolution and 256 shades of gray. For each image, we found the $x, y$ coordinates of the fish in both the direct and reflected parts of the image by using an imageprocessing program (REGIST) developed by one of the authors (P.P.). REGIST is only necessary for digitizing images; for each image, the user, using a mouse, defines the points that correspond to the targets in the direct 
Table 1

Accuracy (in centimeters) of the Proposed Method

\begin{tabular}{lccc}
\hline \multirow{2}{*}{$\begin{array}{l}\text { Measurement } \\
\text { Axis }\end{array}$} & $\begin{array}{c}\text { Accuracy } \\
\text { Standard } \\
\text { Deviation }\end{array}$ & $\begin{array}{c}\text { Mean } \\
\text { Error }\end{array}$ & $\begin{array}{c}\text { No. of } \\
\text { Observations }\end{array}$ \\
\hline$x$ & 0.4 & 0.7 & 77 \\
$y$ & 0.7 & 1.0 & 77 \\
$z$ & 0.3 & 0.6 & 77 \\
Random & 0.5 & 0.7 & 65 \\
$x$ calibrated & 0.2 & 0.5 & 77 \\
$y$ calibrated & 0.3 & 0.3 & 77 \\
\hline
\end{tabular}

Note-Random means the accuracy of 3-D distances between 65 randomly chosen pairs of benchmarks; $x$ and $y$ calibrated are the results of inaccuracy of compensating the calculations for the water refraction.

image (right part) and in the reflected one (left part; see Figure 1). These points (four points for each target point) are then saved in a file for use in the spreadsheet. Although not as simple, it is possible to do the same job using Microsoft Video for Windows (i.e., the Creative Lab's frame grabber Video Blaster) to digitize the images and put them on the Windows clipboard, then to Paintbrush, to extract the coordinates of the targets.

All of the above formulas were placed in a Lotus 1-2-3 spreadsheet, so that after reading the file with the image coordinates, the spreadsheet immediately presented the $x, y, z$ coordinates of the target.

It can be seen from Table 1 that the errors in standard deviations are $0.8 \%-1.5 \%$, compared with the dimensions of the aquarium: $y=46.5 \mathrm{~cm}$ (width), $x=39 \mathrm{~cm}$ (depth), $z=35.5 \mathrm{~cm}$ (height). These errors can be further reduced if the 3-D coordinates are corrected with respect to the effect of water refraction. Since the errors due to the water refraction are systematic, we can compensate by using a linear regression between the true and the calculated coordinates. Applying the parameters of the regression line as calibration coefficients, one corrects the water refraction effect. As can be seen from Table 1, this procedure reduced the errors to $0.5 \%-0.7 \%$ in standard deviations and to $0.6 \%-1.2 \%$ in mean error.

\section{Discussion}

On the basis of the 3-D coordinates, one can easily access several parameters concerning the movement paths of a fish, such as net movement, gross movement, swimming speed, average turning angle, and the fractal dimension of the path (Coughlin et al., 1992). We are using this method for studying the swimming behavior of the Mozambique tilapia (Oreochromis mossambicus, Peters, 1852).
Although some authors note that the use of mirrors could affect fish behavior (Pitcher, 1975), we could not detect any reaction of the fish to the mirror. This fact may be related to the position of the mirror and the differences in illumination between the interior and exterior of the aquarium.

The use of a color video system allows the individual tracking of different fish in a group more easily than with the traditional stereophotographic methods, since one can use frame-by-frame video analysis and colortagging methods to recognize each individual animal. The proposed method is theoretically as accurate as that in which a two-camera system is used, but has the advantage of using a single video camera, with all the 3-D coordinate information in only one image.

\section{REFERENCES}

Coughlin, D. J., Strickler, J. R., \& Sanderson, B. (1992). Swimming and search behaviour in clownfish, Amphiprion perideraion, larvae. Animal Behaviour, 44, 427-440.

Cullen, J. M., SHAw, E., \& Baldwin, H. A. (1965). Methods for measuring the three-dimensional structures of fish schools. Animal Behaviour, 13, 534-543.

DAHMEN, H.-J., \& ZEIL, J. (1984). Recording and reconstructing threedimensional trajectories: A versatile method for the field biologist. Proceedings of the Royal Society of London: Series B, 222, 107-113.

Dambach, M. (1963). Vergleichende Untersuchungen Über das Schwarmverhalten von Tilapia-Jungfischen (Cichlidae, Teleostei). Zeitschrift für Tierpsychologie, 20, 267-296.

Dill, L. M., Dunbrack, R. L., \& Major, P. F. (1981). A new stereophotographic technique for analyzing the three-dimensional structure of fish schools. Environmental Biology of Fishes, 6, 7-13.

HaRrington, S. (1987). Computer graphics: A programming approach (2nd ed.). New York: McGraw-Hill International Editions.

Partridge, B. L., Pitcher, T. J., Cullen, J. M., \& Wilson, J. (1980). The three-dimensional structure of fish schools. Behavioral Ecology \& Sociobiology, 6, 277-288.

Pitcher, T. J. (1973). The three-dimensional structure of schools in the minnow, Phoxinus phoxinus (L.). Animal Behaviour, 21, 673686.

PITCHER, T. J. (1975). A periscopic method for determining the threedimensional positions of fish in schools. Journal of Fisheries Research Board of Canada, 32, 1533-1538.

SYMONS, P. E. K. (1971a). Estimating distances between fish schooling in an aquarium. Journal of Fisheries Research Board of Canada, 28, 1805-1806.

SYMONS, P. E. K. (1971b). Spacing and density in schooling threespine sticklebacks (Gasterosteus aculeatus) and mummichog (Fundulus heteroclistus). Journal of Fisheries Research Board of Canada, 28, 999-1004.

WoLF, P. R. (1985). Elements of photogrammetry, with air photo interpretation and remote sensing (2nd ed.). New York: McGrawHill.

(Manuscript received November 10, 1993; revision accepted for publication June 2, 1994.) 